TRANSACTIONS OF THE

AMERICAN MATHEMATICAL SOCIETY

Volume 354, Number 7, Pages 2921-2932

S 0002-9947(02)02959-8

Article electronically published on March 6, 2002

\title{
ON THE DIMENSIONAL STRUCTURE OF HEREDITARILY INDECOMPOSABLE CONTINUA
}

\author{
ROMAN POL AND MIROSŁAWA REŃSKA
}

\begin{abstract}
Any hereditarily indecomposable continuum $X$ of dimension $n \geq 2$ is split into layers $B_{r}$ consisting of all points in $X$ that belong to some $r$ dimensional continuum but avoid any non-trivial continuum of dimension less than $r$. The subjects of this paper are the dimensional and the descriptive properties of the layers $B_{r}$.
\end{abstract}

\section{Introduction}

Our terminology follows Kuratowski Kur. We consider only separable metrizable spaces and by a compactum we mean a compact space. The main objective of this paper is a proof of Theorem 1.1 about the dimensional structure of hereditarily indecomposable continua, i.e., continua $X$ such that for any pair of intersecting continua $A, B$ in $X$, either $A \subset B$ or $B \subset A$.

R. H. Bing [Bi] constructed hereditarily indecomposable continua of all finite dimensions. The structure of such continua is highly complicated, but in many respects, hereditarily indecomposable continua are generic objects, cf. Krasinkiewicz Kra2, Levin [Lev]. A comprehensive treatment of the subject is given by Lewis Lew.

Bing pointed out that in any $r$-dimensional hereditarily indecomposable continuum $M$ there are points not belonging to any non-trivial continuum in $M$ with dimension less than $r$, [Bi], Theorem 10, cf. [St], sec.2.

1.1. Theorem. Let $X$ be an n-dimensional hereditarily indecomposable continuum and let $B_{r}$ be the set of all points in $X$ that belong to some $r$-dimensional continuum but avoid any non-trivial continuum of dimension less than $r$, where $1 \leq r \leq n$. Then

$$
\operatorname{dim}\left(B_{n} \cup\left(B_{r} \backslash N\right)\right)=n-(r-1),
$$

for any $N$ with $\operatorname{dim} N \leq 0$.

In particular, $\operatorname{dim} B_{n}=1$ and induction with respect to $\operatorname{dim} X$ yields

$$
\operatorname{dim} B_{r}=n-(r-1),
$$

cf. Remark 4.1.

We also have the following

Received by the editors September 5, 2000 and, in revised form, October 5, 2001.

1991 Mathematics Subject Classification. Primary 54F15, 54F45, 54H05.

Key words and phrases. Hereditarily indecomposable continua, dimension, Borel sets. Research partially supported by KBN grant 5 P03A 02420.

(C)2002 American Mathematical Society 
1.2. Corollary. Let $X$ and $B_{n}$ be as in Theorem 1.1. Then for any $(n-2)$ dimensional $\sigma$-compact set $C$ in $X, \operatorname{dim}\left(B_{n} \backslash C\right)=1$.

The sets $B_{r}$, for $r \geq 2$, are never $G_{\delta \sigma}$-sets, being always $G_{\delta \sigma \delta}$-sets in $X$, cf. Proposition 5.1.

For any $r$ with $1<r<n$, there is a point $b \in B_{r}$ and $\epsilon>0$ such that no non-trivial connected set in $X \backslash B_{1}$ of diameter less than $\epsilon$ touches $b$, cf. sec.6 (let us notice that if $X$ is an $n$-Cantor manifold, $B_{r}$ is connected). This yields some refinements of the remarkable fact established by Bing [Bi] that $n$-dimensional hereditarily indecomposable continua $X$ with $n \geq 2$ are not homogeneous, cf. [Ro].

Our proof of Theorem 1.1 is based on some ideas from vM-P], the main tools being "diagonal continua" described in the next section. The origins of such constructions go back to Mazurkiewicz [Ma and Knaster [ $\mathrm{Kn}$ ].

We shall derive Theorem 1.1 from Proposition 3.1, concerning arbitrary compacta, whose subject is related to the work of B. Kaufmann Kau1, Kau2, cf. Remark 3.2 and Comment 7.1.

\section{The DiAgonal CONTINUA}

In this section, $X$ is a fixed $n$-dimensional continuum and

$$
p: X \times I \rightarrow X, q: X \times I \rightarrow I, p(x, t)=x, q(x, t)=t
$$

are the projections from the cylinder, $I$ being the unit interval.

(A). We shall obtain in this section an extension of Proposition B in sec.2 of Po2, providing "diagonal continua" in the cylinders, which we shall use in the proof of Theorem 1.1. Similar continua, but with properties falling short of our needs are also defined in Krasinkiewicz [Kra1], Corollary 3.4 and in [Po1], Corollary 5.2 (i).

2.1. Proposition. Let $X$ be an $n$-dimensional continuum. There exists a continuum $K$ in $X \times I$ joining the top and the bottom of the cylinder such that $\operatorname{dim} E=n$ for any $E \subset K$ whose projection onto I contains all irrationals from a non-trivial interval.

The proof of Proposition 2.1 is given in (B).

We shall need a refined version of a classical theorem of Mazurkiewicz, cf. [Kur], $\S 59$, II, [En], 1.8.18, which follows from a theorem of Holsztyński [Ho], cf. [Mor], Theorem 5.2 .

Theorem. For any $M \subset X \times I$, with $\operatorname{dim} M \leq n-1$ there is a continuum $K \subset X \times I$ missing $M$ and hitting both $X \times\{0\}$ and $X \times\{1\}$.

For the reader's convenience we shall recall briefly a justification of this fact. The argument will be also useful in sec.6.

Let $\left(A_{1}, B_{1}\right), \ldots,\left(A_{n}, B_{n}\right)$ be an essential family in $X$, i.e., $\left(A_{i}, B_{i}\right)$ is a pair of disjoint compacta and for any partitions $L_{i}$ in $X$ between $A_{i}$ and $B_{i}, \bigcap_{i=1}^{n} L_{i} \neq \emptyset$.

Theorem 5.1 in $\left[\mathrm{Ho}\right.$ asserts that the pairs $A_{0}^{*}=X \times\{0\}, B_{0}^{*}=X \times\{1\}$, $A_{i}^{*}=A_{i} \times I, B_{i}^{*}=B_{i} \times I$ for $i=1, \ldots, n$, form an essential family in the cylinder. The proof given by Holsztyński was based on homological arguments, but there is a proof of this fact due to Karno [Kar] using only point-set and simple geometric considerations.

Since $\operatorname{dim} M \leq n-1$, there are partitions $L_{i}$ between $A_{i}^{*}, B_{i}^{*}, i=1, \ldots, n$, such that $L=\bigcap_{i=1}^{n} L_{i}$ is disjoint from $M$. By the essentiality, any partition in $X \times I$ 
between $A_{0}^{*}$ and $B_{0}^{*}$ hits $L$. Therefore, $L$ contains a continuum $K$ joining $A_{0}^{*}$ with $B_{0}^{*}$, cf. [R-S-W], sec.3.

(B). Passing to the proof of Proposition 2.1, let us choose in the set of irrationals in $I$ pairwise disjoint Cantor sets $T_{1}, T_{2}, \ldots$ such that each non-degenerate interval in $I$ contains some $T_{k}$.

Using universal sets from Po1, 3.1, one gets for each $k$ an $(n-1)$-dimensional set $M_{k} \subset X \times T_{k}$, such that any $E \subset X \times I \backslash M_{k}$ with $q(E) \supset T_{k}$ is at least $n$-dimensional. The union $\bigcup_{k=1}^{\infty} M_{k}$ is $(n-1)$-dimensonal, $M_{k}$ being closed in $M$. Therefore, using (A), we get a continuum $K$ in $X \times I$ joining the top and the bottom of the cylinder and missing $M$.

If $E \subset K$ and $q(E)$ contains all irrationals from a non-trivial interval in $I$, then $T_{k} \subset q(E)$ for some $k$, and we infer that $\operatorname{dim} E \geq n$, as $E \cap M_{k}=\emptyset$. This argument shows also that $\operatorname{dim}((\{x\} \times I) \cap K) \leq 0$ for any $x \in X$, i.e., the map $p$ restricted to $K$ is light.

In particular, $\operatorname{dim} K \leq n$, cf. Kur], $\S 45, \mathrm{VI}$, which completes the proof of Proposition 2.1.

\section{A pRoposition CONCERNING THE DIMENSIONAL STRUCTURE OF COMPACTA}

We say, that a point $x$ in the space $X$ is the limit of a sequence $L_{1} \supset L_{2} \supset \ldots$ of sets if for any neighbourhood $V$ of $x$ in $X$ there is $n$ with $x \in L_{n} \subset V$.

3.1. Proposition. Let $X$ be an n-dimensional compactum and let $C_{r}(X)$ be the set of all points in $X$ that are limits of decreasing sequences of $r$-dimensional continua, where $1 \leq r \leq n$. Then $\operatorname{dim}\left(C_{n}(X) \cup\left(C_{r}(X) \backslash N\right)\right) \geq n-(r-1)$ for any $N$ with $\operatorname{dim} N \leq 0$.

3.2. Remark. The case $r=1$, i.e., the fact that $\operatorname{dim}(X \backslash N)=n$ for any zerodimensional $N$ disjoint from $C_{n}(X)$, follows from a statement in the paper by Boris Kaufmann [Kau2] (the other extreme case $r=n$, i.e., the inequality $\operatorname{dim} C_{n}(X) \geq 1$, cf. Kau1, is an easy consequence of this fact). We shall discuss the work of Kaufmann in more detail in Comment 7.1.

3.3. Remark. For any $n$-dimensional compact space $X$ there is an $n$-dimensional hereditarily indecomposable continuum $Z$ and a light map $f: Z \rightarrow X$, cf. [L-S, Theorem 1.2, [Kra3], Corollary 5.2. Therefore, the case $r=1$ in Proposition 3.1 can be derived from the seemingly more special corresponding part of Theorem 1.1.

Proof of Proposition 3.1. In part (A) of the proof we establish some basic properties of the "diagonal continua" described in Proposition 2.1. Then, in step (B), some of these properties are used to demonstrate the case $r=n$ of the assertion of Proposition 3.1. Finally, in step (C), the results of (A) and (B) are combined to get the assertion for arbitrary $r$.

For any compactum $Y, C_{r}(Y)$ is the set defined in Proposition 3.1 (where $X=$ $Y)$.

(A) We shall consider the continuum $K$ in the cylinder $X \times I$, described in Proposition 2.1. We shall denote by

$$
p_{K}: K \rightarrow X, q_{K}: K \rightarrow I
$$

the restrictions to $K$ of the corresponding projections from the cylinder, cf. sec. 2 . As was established at the end of sec. 2, 
(1) $\quad p_{K}$ is light.

Let $P$ be the set of irrationals from $I$, and let, for $r=0,1, \ldots, n$,

(2) $P_{r}=\left\{t \in P: \quad \operatorname{dim} q_{K}^{-1}(t)=r\right\}, P_{\geq r}=P_{r} \cup \ldots \cup P_{n}$.

We shall check that

(3) $\quad p_{K}\left(q_{K}^{-1}\left(P_{0}\right)\right) \subset C_{n}(X)$.

To this end, let us consider $u \in q_{K}^{-1}(t)$ with $t \in P_{0}$ and let $L$ be a non-trivial continuum in $K$ containing $u$. Since $q_{K}^{-1}(t)$ is zero-dimensional, $q_{K}(L)$ is a nontrivial interval in $I$, and the basic property of $K$ yields $\operatorname{dim} L=n$. Since $K$ is a continuum, $u$ is the limit of a sequence $L_{1} \supset L_{2} \supset \ldots$ of non-trivial continua in $K$. Since $\operatorname{dim} L_{j}=n$ we infer from (1) that also $\operatorname{dim} p_{K}\left(L_{j}\right)=n$, cf. [Kur], §45, VI. In effect, $p_{K}(u)$ is the limit of the sequence $p_{K}\left(L_{1}\right) \supset p_{K}\left(L_{2}\right) \supset \ldots$ of $n$-dimensional continua, hence $p_{K}(u) \in C_{n}(X)$.

Let us turn now to another key property of the continuum $K$. We shall verify that for any $E \subset K$ and $r=1, \ldots, n$,

(4) if $q_{K}(E)=P_{0} \cup P_{\geq r}$ then $\operatorname{dim} E \geq n-(r-1)$.

For $r=1$, we assert that $q_{K}(E)=P$ implies $\operatorname{dim} E=n$, which is a special instance of the basic property of $K$.

For $r \geq 2$, we shall derive (4) from this property of $K$ using an idea from vM-P. Let $G=q_{K}^{-1}\left(P_{1} \cup \ldots \cup P_{r-1}\right)$. Then $\operatorname{dim} G \leq r-1$, by (2), cf. [Kur], $\S 45$, VI, and let $Z$ be a zero-dimensional set such that $H=G \backslash Z$ is at most $(r-2)$-dimensional, cf. [Kur], $§ 27$, I. Since $r \geq 2, q_{K}^{-1}(t) \backslash Z \neq \emptyset$, for any $t \in P_{1} \cup \ldots \cup P_{r-1}$, cf. (2). In effect, we get $H \subset K$ such that

(5) $\quad q_{K}(H)=P_{1} \cup \ldots \cup P_{r-1}$ and $\operatorname{dim} H \leq r-2$.

Let $E$ satisfy the assumption in (4). Then $E \cup H \subset K, q_{K}(E \cup H)=P$, cf. (5), and the basic property of $K$ yields $\operatorname{dim}(E \cup H)=n$. This, combined with the addition formula $\mathrm{Kur}, \S 27, \mathrm{I}$, shows that $n \leq \operatorname{dim} E=(r-2)+1$ which is the assertion in (4).

(B) We shall now confirm that the assertion of Proposition 3.1 holds true for $r=n$, i.e., we shall verify that

(6) $\quad \operatorname{dim} C_{n}(X) \geq 1$.

We shall start from the observation that

(7) $\quad C_{n}(t)=C_{n}\left(q_{K}^{-1}(t)\right) \neq \emptyset$, for $t \in P_{n}$.

Indeed, $q_{K}^{-1}(t)$ with $t \in P_{n}$ contains an $n$-dimensional continuum $L_{1}$, cf. (2), and one can choose inductively $n$-dimensional continua $L_{1} \supset L_{2} \supset \ldots$ with diameters tending to zero. The point in the intersection $\bigcap_{j} L_{j}$ belongs to $C_{n}(t)$.

Let us consider the set

(8) $E=q_{K}^{-1}\left(P_{0}\right) \cup \bigcup\left\{C_{n}(t): t \in P_{n}\right\}$.

Then (7) implies $q_{K}(E)=P_{0} \cup P_{n}$ and (4), with $r=n$, shows that

(9) $\quad \operatorname{dim} E \geq 1$.

Let us notice that $p_{K}$ embeds each set $C_{n}(t)$ with $t \in P_{n}$ into $C_{n}(X)$ and therefore, by (8) and (3),

(10) $\quad p_{K}(E) \subset C_{n}(X)$.

By (1), $\operatorname{dim} E \leq \operatorname{dim} p_{K}(E)$, cf. [Kur, $\S 45$, VI, and hence (6) is a consequence of (9) and (10).

(C) We shall now fix an arbitrary $r \leq n$ and a set $N \subset X$ such that

(11) $\operatorname{dim} N=0$ and $N \cap C_{n}(X)=\emptyset$.

By (3), we have also

(12) $\quad N \times I \cap q_{K}^{-1}\left(P_{0}\right)=\emptyset$. 
We have established in (B) that for any $r$-dimensional compactum $Y, \operatorname{dim} C_{r}(Y)$ $\geq 1$. Since any compactum $q_{K}^{-1}(t)$ with $t \in P_{\geq r}$ contains an $r$-dimensional compactum, cf. (2), setting

(13) $\quad C_{r}(t)=C_{r}\left(q_{K}^{-1}(t)\right)$,

we infer that

(14) $\quad \operatorname{dim} C_{r}(t) \geq 1$ for $t \in P_{\geq r}$.

Let us consider the set

(15) $\quad E=\left(q_{K}^{-1}\left(P_{0}\right) \cup \bigcup\left\{C_{r}(t): t \in P_{\geq r}\right\}\right) \backslash N \times I$.

Since $N \times I \cap q_{K}^{-1}(t)$ is at most zero-dimensional, cf. (11), property (14) implies that $C_{r}(t) \backslash N \times I \neq \emptyset$ for any $t \in P_{\geq r}$. This and (12) yield $q_{K}(E)=P_{0} \cup P_{\geq r}$ and, by (4), we conclude that

(16) $\operatorname{dim} E \geq n-(r-1)$.

We shall complete the proof repeating the reasoning at the end of (B). The projection $p_{K}$ embeds each $C_{r}(t)$ with $t \in P_{\geq r}$ into $C_{r}(X)$, and therefore $p_{K}(E) \subset$ $C_{n}(X) \cup\left(C_{r}(X) \backslash N\right)$, cf. (3) and (15). Since $p_{K}$ is $\operatorname{light,} \operatorname{dim} E \leq \operatorname{dim} p_{K}(E)$ and the reference to (16) ends the proof of Proposition 3.1.

\section{Proof of Theorem 1.1}

Let $X$ be an $n$-dimensional compactum, all of whose subcontinua are indecomposable, and let $B_{r}$ be the sets defined in Theorem 1.1.

We shall get the inequality

(1) $\operatorname{dim}\left(B_{n} \cup\left(B_{r} \backslash N\right)\right) \geq n-(r-1)$,

where $\operatorname{dim} N \leq 0$, by making sure that $B_{r} \supset C_{r}(X)$, where $C_{r}(X)$ is the set considered in Proposition 3.1 (in fact, $B_{r}=C_{r}(X)$, cf. sec. 5(B)).

To this end, let $x \in C_{r}(X)$ be the limit of a sequence $L_{1} \supset L_{2} \supset \ldots$ of $r$ dimensional continua. Let $C$ be any non-trivial continuum in $X$ containing $x$ and let $L_{n}$ have diameter smaller than the diameter of $C$. The indecomposability of the continuum $C \cup L_{n}$ implies $L_{n} \subset C$ and hence $\operatorname{dim} C \geq r$. This shows that $x \in B_{r}$.

It remains to justify the inequality

(2) $\operatorname{dim}\left(B_{n} \cup B_{n-1} \cup \ldots \cup B_{r}\right) \leq n-(r-1), r=1, \ldots, n$.

This is evident for $r=1$, so let us fix $r$ with $2 \leq r \leq n$. Let $H_{j}$ be the compact sets in $X$ such that $H=\bigcup_{j=1}^{\infty} H_{j}$ is $(r-1)$-dimensional and $\operatorname{dim}(X \backslash H) \leq n-r$, cf. $\mathrm{Kur}, \S 27, \mathrm{I}$.

Let $L$ be the union of all non-trivial continua in $H$ and $E=H \backslash L$. Then $E \cap H_{j}$ misses all non-trivial continua in $H_{j}$, hence $\operatorname{dim}\left(E \cap H_{j}\right) \leq 0$, cf. [Kur], $\S 47$,VI. By the sum theorem [Kur], $\S 26$,III, $E=\bigcup_{j=1}^{\infty}\left(E \cap H_{j}\right)$ is at most zero-dimensional.

For $j \geq r, B_{j}$ being disjoint from any continuum with dimension less than $r$, we have $B_{j} \cap L=\emptyset$, i.e., $B_{j} \subset(X \backslash H) \cup E$. It follows that $\operatorname{dim}\left(B_{n} \cup \ldots \cup B_{r}\right) \leq(n-r)+1$, cf. Kur], $\S 27, \mathrm{I}$, and we get (2), completing the proof of Theorem 1.1.

4.1. Remark. We have also

(3) $\operatorname{dim} B_{r}=n-(r-1)$.

Theorem 1.1 yields (3) with $r=n$ and the inequality " $\leq$ " follows from (2). We shall check the inequality " $\geq$ " in (3) inductively with respect to the dimension of the space $X$. Assume this is true for all hereditarily indecomposable continua with dimension less than $n$ and let $\operatorname{dim} X=n$. One can assume that $X$ is an $n$-Cantor manifold [Kur], $\S 46$, XI. Let $Z$ be any partition of $X$. Then there is an $(n-1)$ dimensional continuum $Y \subset Z$ and by the inductive assumption, $\operatorname{dim}\left(Y \cap B_{r}\right) \geq$ $(n-1)-(r-1)$. 
It follows that any partition in $X$ hits $B_{r}$ in at least an $(n-(r-1)-1)$-dimensional set, and hence $\operatorname{dim} B_{r} \geq n-(r-1)$, cf. [Kur], §27,II.

4.2. Proof of Corollary 1.2. Let $X$ and $C$ be as in Corollary 1.2. Aiming at a contradiction, assume that $\operatorname{dim}\left(B_{n} \backslash C\right) \leq 0$. Then there is a countable base in $X$ whose elements have boundaries disjoint from $B_{n} \backslash C$. Let $L$ be the union of these boundaries. Then

(4) $\quad L$ is $\sigma$-compact, $L \cap B_{n} \subset C$, and $\operatorname{dim}(X \backslash L) \leq 0$.

Let us check that

$$
\operatorname{dim} L \leq n-1 .
$$

To this end, let us consider any continuum $K \subset L$ and let $K_{1}$ be a $\sigma$-compact subset of $K$ with $\operatorname{dim} K_{1}<\operatorname{dim} K$ and $\operatorname{dim}\left(K \backslash K_{1}\right) \leq 0$. The sets $C$ and $K_{1}$ being $\sigma$-compact, $K_{2}=K_{1} \cup C$ is at most $(n-1)$-dimensional. Since $B_{n} \cap K \subset K_{2}$, cf. (4), $\operatorname{dim}\left(K \backslash K_{2}\right) \leq 0$ and $\operatorname{dim} K_{2} \leq n-1$, we conclude that $\operatorname{dim} K \leq n-1$ using Theorem 1.1. This demonstrates (5), $L$ being $\sigma$-compact.

Let $L_{1}$ be a $\sigma$-compact subset of $L$ with

and let

Then, by (4) and (6),

$$
\operatorname{dim} L_{1} \leq n-2 \text { and } \operatorname{dim}\left(L \backslash L_{1}\right) \leq 0,
$$

$$
L_{2}=L_{1} \cup C, N=L \backslash L_{2} .
$$

We have also $\operatorname{dim} L_{2} \leq n-2, L_{1}$ and $C$ being $\sigma$-compact, cf. (6) and (7). In effect, (4) and (7) yield $\operatorname{dim}(X \backslash N) \leq \operatorname{dim}(X \backslash L)+\operatorname{dim} L_{2}+1 \leq n-1$. But this, combined with (8), contradicts Theorem 1.1.

\section{The Descriptive COMPleXity of the Sets $B_{r}$}

5.1. Proposition. Let $X$ be an $n$-dimensional hereditarily indecomposable continuum, and let $B_{r}$ be the sets defined in Theorem 1.1. Then

(i) each $B_{r}$ is a $G_{\delta \sigma \delta}$-set in $X$,

(ii) for $r \geq 2, B_{r}$ is not a $G_{\delta \sigma}$-set in $X$.

5.2. Remark. The essential part of (ii) is that no $B_{n}$ is a $G_{\delta \sigma}$-set in $X$ provided $\operatorname{dim} X=n \geq 2$. Indeed, if we know this is true for any $n \geq 2$, then given $r$ with $2 \leq r \leq n$, we can use this information to conclude that $B_{r} \cap Y$ is not a $G_{\delta \sigma}$-set in $Y$ for any $r$-dimensional continuum $Y$ in $X$, and hence $B_{r}$ is not a $G_{\delta \sigma}$-set in $X$.

(A) We shall prove part (i) of the assertion using a theorem of Kelley [Kel], Theorem 8.5, based on Whitney's maps, to the effect that for any $k$ there is an open mapping

$$
\pi_{k}: X \rightarrow W_{k}
$$

of the hereditarily indecomposable continuum $X$ whose fibers $\pi_{k}^{-1}(t)$ are continua with

$$
0<\delta_{k}<\operatorname{diam} \pi_{k}^{-1}(t)<\frac{1}{k} .
$$

Passing to the proof of (i), let us fix $r$. Setting

$$
W_{k, j}=\left\{w \in W: \operatorname{dim} \pi_{k}^{-1}(w) \leq j\right\},
$$

we shall check that

$$
B_{r}=\bigcup_{k=1}^{\infty} \pi_{k}^{-1}\left(W_{k, r}\right) \backslash \bigcup_{k=1}^{\infty} \pi_{k}^{-1}\left(W_{k, r-1}\right) .
$$


Let $x \in B_{r}$. Then $\pi_{k}(x) \notin W_{k, r-1}$ for any $k$. On the other hand, $x$ belongs to an $r$-dimensional continuum $C$, and let $k$ be such that $\frac{1}{k}$ is smaller than the diameter of $C$. Let $D=\pi_{k}^{-1}\left(\pi_{k}(x)\right)$. Since $X$ is hereditarily indecomposable, $D \subset C$, hence $\operatorname{dim} D \leq r$. In effect, $\pi_{k}(x) \in W_{k, r}$.

This demonstrates the inclusion " $\subset$ " in (1), and the opposite one is transparent.

Each $W_{k, j}$ is a $G_{\delta \sigma}$-set in $W_{k}$, cf. [Kur, $\S 45$, IV, Theorem 4 , hence part (i) of the assertion follows from formula (1).

(B) By Remark 5.2, to check part (ii) of the assertion it is enough to concentrate on the sets $B_{n}$.

Remark 4.1 guarantees that

(2) $\operatorname{dim}\left(B_{1} \cap L\right)=\operatorname{dim} L$ for any non-trivial continuum $L$ in $X$.

Let us consider arbitrary sets $G_{j}, j=1,2, \ldots$, such that

(3) $G_{j} \subset B_{n}$ and $G_{j}$ is a $G_{\delta}$-set in $X$.

We have to check that

(4) $\quad B_{n} \backslash \bigcup_{j} G_{j} \neq \emptyset$.

To this end we shall choose inductively $n$-dimensional continua $L_{1} \supset L_{2} \supset \ldots$ with

(5) $\quad L_{j} \cap G_{j}=\emptyset$ and $\operatorname{diam} L_{j} \leq \frac{1}{j}$,

where diam stands for the diameter of the set. To start the process, we let $G_{0}=\emptyset$, $L_{0}=X$.

Assume that $L_{j}$ is already defined. Since $B_{1} \cap B_{n}=\emptyset, \operatorname{dim}\left(L_{j} \backslash G_{j+1}\right) \geq$ $\operatorname{dim}\left(B_{1} \cap L_{j}\right)=n$, cf. (2) and (3). Since $L_{j} \backslash G_{j+1}$ is $\sigma$-compact, we can pick an $n$-dimensional continuum $L_{j+1} \subset L_{j} \backslash G_{j+1}$, and we can demand in addition that $\operatorname{diam} L_{j+1} \leq \frac{1}{j+1}$.

Let $x$ be the limit point of the sequence $L_{1} \supset L_{2} \supset \ldots$, cf. sec. 3. For any continuum $C$ in $X$ containing $x$, if $\frac{1}{j}<\operatorname{diam} C$ then $L_{j} \subset C$, by the hereditary indecomposability of $X$, and hence $\operatorname{dim} C=n$. It follows that $x \in B_{n}$. But also $x \in \bigcap_{j} L_{j} \subset X \backslash \bigcup_{j} G_{j}$, cf. (5), and we get (4).

\section{Points in $B_{r}$ That ARE AVOIded By ANy SMALL NON-TRIVIAL CONNECTED SETS IN $B_{r}$}

The results of this section refine the remarkable fact pointed out by Bing [Bi], sec. 6 , that $n$-dimensional hereditarily indecomposable continua with $n \geq 2$ are not homogeneous; cf. also Rogers [Ro]. We adopt the notation from Theorem 1.1.

6.1. Theorem. Let $X$ be an $n$-dimensional hereditarily indecomposable continuum and $1<r<n$. There is a point $b \in B_{r}$ and its neighbourhood $V$ in $X$ such that any non-trivial continuum in $V$ touching $b$ is separated by a relatively open 1-dimensional set missing $b$.

The point $b$ and its neighbourhood $V$ described in this theorem substantiate the title of this section. Indeed, let $C$ be any connected set in $B_{r}$ whose closure $L$ is contained in $V$ and $b \in C$. Then the continuum $L$ is either the singleton $\{b\}$ or there is a relatively open $G$ in $L$ with $\operatorname{dim} G=1$ separating $L$. But the second case is impossible, as such $G$ would be disjoint from $B_{r}$, and hence it would provide a decomposition of the connected $C$ into two disjoint nonempty closed sets.

6.2. Corollary. Let $X$ and $b \in B_{r}$ be as in Theorem 6.1 and let $c$ be the limit of n-Cantor manifolds in $X$. Then there is no embedding $h: B_{r} \cup\{c\} \rightarrow X$ with $h(c)=b$. 
Before giving proofs of these facts, let us make the following

6.3. Remark. Let $X$ and $r$ be as in Theorem 6.1 and let $M$ be an $m$-Cantor manifold in $X$ with $m>r$. Then the set $M \cap B_{r}$ is connected and all its nonempty relatively open sets have dimension $m-(r-1)$.

The connectedness of $M \cap B_{r}$ follows from the fact that any partition in $M$ has dimension at least $r$, and therefore it must hit $B_{r}$. The second part follows from Remark 4.1.

Using this remark, we get Corollary 6.2 from Theorem 6.1 as follows. Assume on the contrary that the embedding $h$ in Corollary 6.2 exists, let $V$ be a neighbourhood of $b$ in $X$ described in Theorem 6.1, and let $M$ be an $n$-Cantor manifold in $X$ with the closure $L$ of $h\left(M \cap B_{r}\right)$ contained in $V$. Then $L$ must be the singleton $\{b\}$. Otherwise $L$, and hence $M \cap B_{r}$, would contain a relatively open 1-dimensional set, which is impossible as $n-(r-1)>1$.

Let us notice that, slightly changing the argument, one can also conclude that for any continuous map $f: B_{r} \cap\{c\} \rightarrow X \backslash B_{1}$ with $f(c)=b, \operatorname{dim} f^{-1}(b)=n-(r-1)$.

Theorem 6.1 is a direct consequence of Lemma 6.5 concerning arbitrary compacta. Our justification of this lemma follows closely some arguments used in the proof of Proposition 3.1, and therefore certain details will be omitted. We shall need the following fact.

6.4. Lemma. Let $p: S \rightarrow I$ be a continuous map of a compactum $S$ onto the interval, and let $T$ be a Cantor set in $I$. For each $r$ there is a set $M \subset p^{-1}(T)$ with $\operatorname{dim} M \leq r-1$ such that whenever $E \subset S \backslash M$ and $T \subset p(E)$, then $\operatorname{dim} E \geq r$.

Indeed, first embedding $S$ in the Hilbert cube $I^{\infty}$ and then identifying $S$ with the graph of $p$, one can assume that $S \subset I \times I^{\infty}$ and that $p$ is the projection onto $I$ restricted to $S$. Then one can take as $M$ the trace on $S$ of a universal $(r-1)$-dimensional set constructed in [Po2], cf. sec.2 (B).

6.5. Lemma. Any $(r+1)$-dimensional continuum $S, r \geq 2$, contains a non-trivial continuum $K$ and a point $b \in K$ such that any non-trivial continuum $L \subset K$ touching $b$ is r-dimensional and $L$ can be separated by a relatively open 1-dimensional set missing $b$.

Proof. Let $\left(A_{0}, B_{0}\right), \ldots,\left(A_{n}, B_{n}\right)$ be an essential family in $S$, let $T \subset(0,1)$ be a Cantor set, and let $p: S \rightarrow I$ be a continuous mapping with

(1) $\quad A_{0} \subset p^{-1}(0), B_{0} \subset p^{-1}(1)$ and $\operatorname{dim} p^{-1}(t) \leq r, t \in T$,

cf. $\mathrm{vM}-\mathrm{P}$, sec.3 and [R-S-W], sec.2. Let $J_{1}, J_{2}, \ldots$ be an enumeration of the components of $I \backslash T$ and let $T_{1}, T_{2}, \ldots$ be pairwise disjoint Cantor sets in $T$, missing all boundaries bd $J_{k}$, such that any relatively open set in $T$ contains some $T_{k}$.

Using Lemma 6.4 we find $(r-1)$-dimensional sets $M_{i} \subset p^{-1}\left(T_{i}\right)$ such that, for any $E \subset S$,

(2) if $E \cap M_{i}=\emptyset$ and $T_{i} \subset p(E)$, then $\operatorname{dim} E \geq r$.

Let us choose also, for each $k$, a set $H_{k}$ such that

(3) $\quad H_{k} \subset p^{-1}\left(J_{k}\right), \operatorname{dim} H_{k} \leq r-1$ and $\operatorname{dim}\left(p^{-1}\left(J_{k}\right) \backslash H_{k}\right) \leq 1$.

Then, setting

(4) $\quad M=\bigcup_{i=1}^{\infty} M_{i} \cup \bigcup_{k=1}^{\infty} H_{k}$,

we have by the sum theorem [Kur], §27.I,

$$
\operatorname{dim} M \leq r-1,
$$


as $M_{i}$ and $H_{k}$ are relative $F_{\sigma}$-sets in $M$. Since $\operatorname{dim} S-\operatorname{dim} M \geq 2$, there is a continuum $K$ in $S$ joining $A_{0}$ and $B_{0}$, and missing $M$, (cf. sec. $2(\mathrm{~A})$ and $2(\mathrm{~B})$ ). By (2) and (4),

(6) if $E \subset K$ and $T_{i} \subset p(E)$, then $\operatorname{dim} E \geq r$.

Let us notice also that (3) and (4) imply

(7) $\quad \operatorname{dim}\left(K \cap p^{-1}\left(J_{k}\right)\right) \leq 1$.

(8) $\operatorname{dim} K=r$.

Since $\operatorname{dim} p^{-1}(T) \leq r$, by (1), this fact and (2) yield (cf. also (6))

Let $W_{1}, W_{2}, \ldots$ be an open base in $K$ whose elements have at most $(r-1)$ dimensional boundaries $F_{i}=\operatorname{bd} W_{i}$. Then, by $(2)$, the projections $p\left(F_{i}\right)$ have relatively empty interiors in $T$. The Baire Category Theorem allows one to pick a point

(9) $s \in T \backslash\left(\bigcup_{i=1}^{\infty} p\left(F_{i}\right) \cup \bigcup_{k=1}^{\infty}\right.$ bd $\left.J_{k}\right)$.

The choice of $s$ in (9) guarantees that each $W_{i} \cap p^{-1}(s)$ is open-and-closed in $p^{-1}(s) \cap K$, and hence

(10) $\quad \operatorname{dim} p^{-1}(s) \cap K=0$.

We shall check that the continuum $K$ and any $b \in p^{-1}(s) \cap K$ satisfy the assertion of Lemma 6.5.

Let $L$ be an arbitrary non-trivial continuum in $K$ containing $b$. By (10), $p(L)$ is a non-trivial interval in $I$. Since $s \in p(L) \backslash \bigcup_{k=1}^{\infty} \operatorname{bd}\left(J_{k}\right)$, cf. (9), we infer that $p(L)$ contains some $T_{i}$ and that some $J_{k}$ missing $p(s)$ separates $p(L)$. Then $\operatorname{dim} L=r$, by (6) and (8). The open set $p^{-1}\left(J_{k}\right)$ separates $L$, missing $s$, and $\operatorname{dim}\left(L \cap p^{-1}\left(J_{k}\right)\right) \leq 1$, by $(7)$.

Proof of Theorem 6.1. Let $X$ and $r$ be as in Theorem 6.1. Since $X$ contains an $(r+1)$-dimensional continuum $S$ we get the continuum $K$ and $b \in K$ described in Lemma 6.5.

Since any non-trivial continuum in $K$ touching $b$ is $r$-dimensional, we have $b \in$ $B_{r}$. Let $V$ be a neighbourhood of $b$ in $X$ with $K \backslash V \neq \emptyset$, and let $L$ be any non-trivial continuum in $V$ containing $b$. Then, by the indecomposability of the continuum $L \cup K$ we have $L \subset K$ and therefore, by the choice of $K$, the continuum $L$ is separated by a relatively open 1-dimensional set missing $b$.

6.6. Remark. Let $X$ and $B_{r}$ be as in Theorem 6.1. Then no $G_{\delta}$-set $G$ in $X$ containing $B_{r}$ is homogeneous.

Indeed, such $G$ must contain a point $c$ that is the limit of $n$-Cantor manifolds in $X$. To see this, let $G=\bigcap_{j} U_{j}$ with $U_{j}$ open and choose inductively $n$-Cantor manifolds $M_{1} \supset M_{2} \supset \ldots$ with $M_{j} \subset U_{j}$ and the diameters of $M_{j}$ tending to zero. This is possible, as each $X \backslash U_{j}$ is disjoint from $B_{r}$, hence at most $(r-1)$-dimensional, and therefore $U_{j}$ has an $n$-dimensional intersection with any $n$-dimensional set in $X$. Having chosen $M_{j}$, we let $c$ be the point in $\bigcap_{j} M_{j}$.

The non-homogenity of $G$ is an instant consequence of Corollary 6.2.

\section{Comments}

7.1. The papers by Boris Kaufmann [Kau1 and Kau2]. Kaufmann's works seem not to be widely known. We learned about Kau1, Kau2 from P.S. Aleksandrov's book [Al2] only after obtaining the main results of this paper. Aleksandrov credits Kaufmann with substantial contributions to homological dimension theory, pointing out difficulties in following his work, cf. [Al2], p.194, Al1], p.14. 
Kaufmann considers in Kau1 and Kau2 "nuclei" in $n$-dimensional compacta $X \subset R^{m}$ - the $n$-dimensional compacta irreducibly linked with some $(m-n-1)$ cycle in an open ball in $R^{m}$, and distinguishes the set $\Phi^{n}$ of the limit points of descending sequences of nuclei in $X$, calling such points "manifold points of $X$ " (each nucleus is an $n$-Cantor manifold), cf. 7.3. Theorems 2 and 3 in Kau1 assert that any $(n-1)$-dimensional partition $L$ of the nucleus $F$ in $X$ must separate nuclei in $F$ with arbitrarily small diameters, and in effect, the set $F \cap \Phi^{n}$ is connected (this is not true for arbitrary $n$-Cantor manifolds, cf. 7.2). In Kau2], I.3, Kaufmann introduces an inductive notion of "being a set homogeneously $j$-dimensional in $X$ " and Theorem $\Phi^{r}$ in [Kau2], p.34, asserts that $\Phi^{n}$ is homogeneously $n$-dimensional in $X$. One can show that this is equivalent to the statement that $\operatorname{dim}(X \backslash N)=n$ for any $N$ disjoint from $\Phi^{n}$ with $\operatorname{dim} N \leq 0$, cf. Remark 3.2. Let us notice that a generalization of this assertion stated in footnote (11) on p. 19 in [Kau2 and in Kau3, p.247, is not valid - any hereditarily indecomposable cut of the cube $I^{n}$, $n \geq 3$, provides a counterexample.

Kaufmann's reasoning is based on intricate homological arguments.

7.2. An example related to Proposition 3.1. Let $Z$ be an hereditarily indecomposable $n$-Cantor manifold and let $C$ be an $(n-1)$-dimensional continuum in $Z, n \geq 2$.

Let $X$ be the result of matching two copies of $Z$ along the continuum $C$ and let $L$ be the copy of $C$ in $X$. Then $X$ is an $n$-Cantor manifold and $L$ separates $X$. However, $L$ is disjoint from the set $C_{n}(X)$ in Proposition 3.1 and $L$ does not separate any $n$-Cantor manifold in $X$ with diameter smaller than the diameter of $L$.

7.3. A remark related to Proposition 3.1. Let $X$ be an $n$-dimensional compactum and let $\mathcal{M}$ be a collection of $n$-Cantor manifolds in $X$, such that each $n$-Cantor manifold in $X$ contains an element of $\mathcal{M}$.

Let $C(\mathcal{M})$ be the set of points that are limits of decreasing sequences of elements of $\mathcal{M}$. Then, for any $G_{\delta}$ set $G$ in $X$ disjoint from $C(\mathcal{M}), \operatorname{dim}\left(C_{n}(X) \backslash G\right) \geq 1$, where $C_{n}(X)$ is defined in Proposition 3.1.

Indeed, aiming at a contradition, assume that $\operatorname{dim}\left(C_{n}(X) \backslash G\right) \leq 0$ and let $G=G_{1} \cap G_{2} \cap \ldots$, where $G_{1} \supset G_{2} \supset \ldots$ are open. We shall define a sequence $M_{1} \supset M_{2} \supset \ldots$ of elements of $\mathcal{M}$ with $M_{i} \subset G_{i}$ and $\operatorname{diam} M_{i} \leq \frac{1}{i}$. Suppose we have chosen $M_{i}$. By Propositon 3.1, $\operatorname{dim}\left(C_{n}(X) \cap M_{i}\right) \geq 1$, and hence $M_{i} \cap G \neq \emptyset$. Therefore $\operatorname{dim} M_{i} \cap G_{i+1}=n$, as each non-empty open set in $M_{i}$ is $n$-dimensional. Let $N \subset M_{i} \cap G_{i+1}$ be an $n$-Cantor manifold with diam $N \leq \frac{1}{i+1}$. We choose $M_{i+1}$ in $\mathcal{M}$ with $M_{i+1} \subset N$. Having defined the sequence $M_{1} \supset M_{2} \supset \ldots$ let us consider the limit $c$. We get $c \in C(\mathcal{M}) \cap G=\emptyset$, a contradiction.

Let us assume in addition that $X$ is an hereditarily indecomposable continuum. Then $C(\mathcal{M})$ is not a $G_{\delta}$-set in $X$. Indeed, since $C(\mathcal{M}) \cap B_{1}=\emptyset, \operatorname{dim}(X \backslash C(\mathcal{M}))=$ $n$, by Remark 4.1, and on the other hand, each compact set in $X \backslash C(\mathcal{M})$ is at most $(n-1)$-dimensional.

It follows, that the statement in Kau2, sec. 4, that the union $N_{\delta}$ of all $n$ dimensional "nuclei" in $X$ of diameter $<\delta$ is locally compact, is incorrect. In fact, in the hereditarily indecomposable continuum $X, N_{\delta}$ is not completely metrizable for some $\delta$. 
7.4. An example on weakly infinite-dimensional continua. A space $E$ is weakly infinite-dimensional if for each sequence of pairs $\left(A_{i}, B_{i}\right), i=1,2, \ldots$, of disjoint closed sets in $E$ there are partitions $L_{i}$ in $E$ between $A_{i}$ and $B_{i}$ such that $\bigcap_{i} L_{i}=\emptyset$, cf. [En]. The following result was obtained jointly by Elżbieta Pol and the present authors.

Example. There is a weakly infinite-dimensional continuum $X$ such that the set $B_{\infty}$ of points in $X$ avoiding any non-trivial finite-dimensional continuum in $X$ is not weakly infinite-dimensional.

The construction will be included in the paper by Elżbieta Pol and the second author $\left[\mathrm{P}-\mathrm{R}\right.$ concerning the sets $B_{\infty}$ in infinite-dimensional hereditarily indecomposable continua.

Some questions. (A) Let $S$ be an hereditarily indecomposable $(n+1)$-dimensional continuum, $n \geq 2$, and let $K$ be the $n$-dimensional continuum in $S$ described in Lemma 6.5.

Then in $K$, the set $B_{n}$ contains points at which $B_{n}$ is 0-dimensional and also, being 1-dimensional, $B_{n}$ contains points at which it is 1-dimensional, cf. [Kur], $\S 25$, I. Therefore, the space $B_{n}$ in $K$ is not homogeneous.

Let us consider the set $B_{r}$ in an $n$-dimensional hereditarily indecomposable continuum $X$, where $1<r<n$. The results of sec. 6 show that there is a point $b \in B_{r}$ and $\delta>0$ such that no neighbourhood of $b$ in $B_{r}$ of diameter less than $\delta$ is homogeneous.

The assertion of a theorem of Kaufmann discussed in sec. 7.1, combined with the results in sec. 6 , imply that the set $B_{r}$ is not homogeneous. We do not know any other arguments supporting this claim.

(B) Using a method of "condensation of singularities" cf. [Ch-P], Elżbieta Pol and the second author $[\mathrm{P}-\mathrm{R}]$ constructed examples of 2-dimensional hereditarily indecomposable continua $X^{\prime}, X^{\prime \prime}$, such that in $X^{\prime}$ the set $B_{2}$ is residual, while in $X^{\prime \prime}$ the set $B_{1}$ is residual (these possibilities exclude each other, the sets $B_{r}$ being Borel). However, we have no information about the Baire category of the sets $B_{r}$ in hereditarily indecomposable Cantor manifolds.

Let $X$ be an $n$-dimensional hereditarily indecomposable continuum and let $\mathcal{M}$ be the collection of all $n$-Cantor manifolds in $X$. Then the set $C(\mathcal{M})$ defined in sec. 7.4 is projective. What exactly is the descriptive class of $C(\mathcal{M})$ ?

(C) By a theorem of Krasinkiewicz [Kra2] and Levin [Lev], a typical, in the sense of Baire category in function spaces, mapping $f: I^{n} \rightarrow I$ has the property that any non-trivial continuum in $I^{n}$ mapped by $f$ to a point is hereditarily indecomposable. Let $f$ be such a typical mapping and let $B_{r}(f)$ be the set of points in $I^{n}$ that belong to some $r$-dimensional continuum taken by $f$ to a point, but which avoid any non-trivial continuum of dimension less than $r$ mapped to a point. Then, for $1 \leq r \leq n-1$ (let us recall that by a Hurewicz theorem the fibers of a typical map $f: I^{n} \rightarrow I$ are at most $(n-1)$-dimensional, cf. [Kur], $\left.\S 45, \mathrm{IX}\right)$, we get

$$
n-r \leq \operatorname{dim} B_{r}(f) \leq n-(r-1) \text {. }
$$

Is it true that, typically, the upper bound is attained?

\section{REFERENCES}

[Al1] P.S.Alexandroff, On some main directions in general topology, Uspekhi Mat. Nauk 19 (1964), 3-46 (in Russian). 
[Al2] P.S.Alexandroff, Introduction to Homological Dimension Theory, "Nauka", Moscow, 1975 (in Russian).

[Bi] R.H.Bing, Higher-dimensional hereditarily indecomposable continua, Trans. Amer. Math. Soc. 71 (1951), 267-273. MR 13:265c

[Ch-P] V.A.Chatyrko, E.Pol, Continuum many Fréchet types of hereditarily strongly infinitedimensional Cantor manifolds, Proc. Amer. Math. Soc. 128 (2000), 1207 - 1213. MR 2000i:54051

[En] R.Engelking. Theory of Dimensions, Finite and Infinite, Heldermann Verlag, Lemgo, 1995. MR 97j:54033

[Ho] W.Holsztyński, Universality of the product mappings onto products of $I^{n}$ and snake-like spaces, Fund. Math. 64 (1969), 147-155. MR 39:6249

[Kar] Z.Karno, On a theorem of P.S. Aleksandrov, Coll. Math. 72 (1997), 39-51. MR 98b:54047

[Kau1] B.Kaufmann, The dissection of closed sets of arbitrary dimension and the generalized Brouwer-Alexandroff theorem, Proc. Camb. Phil. Soc. 31 (1935), 525-535.

[Kau2] B.Kaufmann, On infinitesimal properties of closed sets of arbitrary dimension, Annals of Math. 38 (1937), 14-35

[Kau3] B.Kaufmann, Of the extension of the Pflastersatz, Proc. Camb. Phil. Soc. 32 (1936), 238-247.

[Kel] J.L.Kelley, Hyperspaces of a continuum, Trans. Amer. Math. Soc. 52 (1942), 22-36. MR 3:315b

[Kn] B.Knaster, Sur les coupures biconnexes des espaces euclidiens de dimension $n>1$ arbitraire, Matem. Sbornik 19 (1946), 9-18. MR 8:164j

[Kra1] J.Krasinkiewicz, Homotopy separators and mappings into cubes, Fund. Math. 131 (1988), 149-154. MR 90i:54081

[Kra2] J.Krasinkiewicz, On mappings with hereditarily indecomposable fibers, Bull. Pol. Acad. Sci. 44 (1996), 147-156. MR 97g:54016

[Kra3] J.Krasinkiewicz, On approximation of mappings into 1-manifolds, Bull. Pol. Acad. Sci. 44 (1996), 431-440. MR 97k:54011

[Kur] K.Kuratowski, Topology, vol. I, Academic Press, New York, 1966, and vol. II, Academic Press, New York, 1968. MR 36:840; MR 41:4467

[Lev] M.Levin, Bing maps and finite-dimensional maps, Fund. Math. 151 (1996), 47-52. MR 97e:54031

[L-S] M.Levin, Y.Sternfeld, Hyperspaces of two-dimensional continua, Fund. Math. 150 (1996), 17-24. MR 97d:54015

[Lew] W.Lewis, The pseudo-arc, Bol. Soc. Mat. Mexicana 5 (1999), 25-77. MR 2000f:54029

[Ma] S.Mazurkiewicz, Sur les problemes $\kappa$ and $\lambda$ de Urysohn, Fund. Math. 10 (1926), 311-319.

[vM-P] J.van Mill and R.Pol, On the existence of weakly $n$-dimensional spaces, Proc. Amer. Math. Soc. 113 (1991), 581-585. MR 91m:54043

[Mor] K.Morita, Čech cohomology and covering dimension for topological spaces, Fund. Math. 87 (1975), 31-52. MR 50:14706

[P-R] E.Pol, M.Reńska, On Bing points in infinite-dimensional hereditarily indecomposable continua, Topology and Appl. (to appear).

[Po1] R.Pol, Countable dimensional universal sets, Trans. Amer. Math. Soc. 297 (1986), 255268. MR 87h:54067

[Po2] R.Pol, An $n$-dimensional compactum which remains $n$-dimensional after removing all Cantor $n$-manifolds, Fund. Math. 136 (1990), 127-131. MR 91j:54061

[Ro] J.T.Rogers, Jr., Orbits of higher-dimensional hereditarily indecomposable continua, Proc. Amer. Math. Soc. 95 (1985), 483-485. MR 86k:54054

[R-S-W] L.R.Rubin, R.M.Schori, and J.J.Walsh, New dimension theory technique for constructing infinite-dimensional examples, General Topology Appl. 10 (1979), 93-102. MR 80e:54049

[St] Y.Sternfeld, On finite-dimensional maps and other maps with "small" fibres, Fund. Math. 147 (1995), 127-133. MR 96e:54030

Institute of Mathematics, University of WarsaW, Banacha 2, 02-097 Warsaw, Poland

E-mail address: pol@mimuw.edu.pl

Institute of Mathematics, University of Warsaw, Banacha 2, 02-097 Warsaw, Poland

E-mail address: mrenska@mimuw.edu.pl 